\title{
Feeling the pull: a new device for sphincter augmentation
}

A new magnetic device augments lower oesophageal sphincter (LES) activity, according to findings published in the New England Journal of Medicine.

Approximately $40 \%$ of patients with GERD have a partial response to PPIs and seek alternative therapies if their quality of life is compromised. The only established treatment option for these patients is surgery, which has limited acceptance owing to the potential of adverse events. Robert Ganz and colleagues are now establishing the safety and effectiveness of a new magnetic device that augments the LES. The aim of this expandable magnetic device is to improve sphincter barrier function without altering normal anatomical structure or interfering with swallowing, belching or vomiting.

"The device involves the use of magnetic attraction through adjacent magnetic beads, which augments the resistance of the oesophageal sphincter to abnormal opening associated with reflux," write the authors. The device is placed around the oesophagus without compressing the underlying muscle and stays in position owing to the permanent attraction between the beads. Wires connect the beads enabling the device to expand during the passage of food.

The researchers have previously shown the feasibility of this approach in a pilot study. Here, they report the 3-year outcomes of a 5-year prospective study of 100 patients with GERD who had this device fitted. Patients were included if they had a $\geq 6$-month history of GERD and had a partial response to PPIs. Implantation of the device was done by surgeons using standard laparoscopic techniques. No control group was included in this study.

The primary outcome of the study was normalized oesophageal acid exposure (or $\geq 50 \%$ reduction in acid exposure at 1 year) and was achieved in $64 \%$ of patients. Secondary outcomes were $\geq 50 \%$ improvement in quality of life related to GERD (achieved in 92\% of patients) and $\geq 50 \%$ reduction in PPI use at 1 year

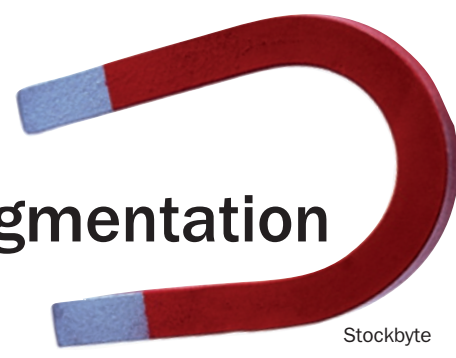

(achieved in 93\% of patients). "In a group of patients with GERD failing PPIs, this device controlled oesophageal acid exposure, and eliminated symptoms and medication usage in nearly all, which is very impressive," explains Ganz.

The investigators then went on to look at the safety of the device. The most frequent adverse event was dysphagia (reported in $68 \%$ of patients postoperatively; this level reduced to only $11 \%$ at 1 year and $4 \%$ at 3 years).

Further studies will be needed to elucidate the long-term safety of this device. "The next step is to directly compare this device against other treatments," adds Ganz.

Katherine Smith

Original article Ganz, R. A. et al. Esophageal sphincter device for gastroesophageal reflux disease. N. Engl. J. Med. $368,719-727$ (2013) 